\title{
Increased Set1 binding at the promoter induces aberrant epigenetic alterations and up-regulates cyclic adenosine 5'-monophosphate response element modulator alpha in systemic lupus erythematosus
}

Qing Zhang ${ }^{1}$, Shu Ding ${ }^{2}$, Huilin Zhang ${ }^{3}$, Hai Long ${ }^{1}$, Haijing Wu' ${ }^{1}$ Ming Zhao ${ }^{1}$, Vera Chan ${ }^{4}$, Chak-Sing Lau ${ }^{4}$ and Qianjin $\mathrm{Lu}^{1 *}$

\begin{abstract}
Background: Up-regulated cyclic adenosine 5'-monophosphate response element modulator a (CREMa) which can inhibit IL-2 and induce IL-17A in T cells plays a critical role in the pathogenesis of systemic lupus erythematosus (SLE). This research aimed to investigate the mechanisms regulating CREMa expression in SLE.

Results: From the chromatin immunoprecipitation (ChIP) microarray data, we found a sharply increased H3 lysine 4 trimethylation (H3K4me3) amount at the CREMa promoter in SLE CD4+ T cells compared to controls. Then, by ChIP and real-time PCR, we confirmed this result. Moreover, H3K4me3 amount at the promoter was positively correlated with CREMa mRNA level in SLE CD4+ T cells. In addition, a striking increase was observed in SET domain containing 1 (Set1) enrichment, but no marked change in mixed-lineage leukemia 1 (MLL1) enrichment at the CREMa promoter in SLE CD4+ T cells. We also proved Set1 enrichment was positively correlated with both H3K4me3 amount at the CREMa promoter and CREMa mRNA level in SLE CD4+ T cells. Knocking down Set1 with siRNA in SLE CD4+ T cells decreased Set1 and H3K4me3 enrichments, and elevated the levels of DNMT3a and DNA methylation, while the amounts of $\mathrm{H} 3$ acetylation $(\mathrm{H} 3 \mathrm{ac})$ and $\mathrm{H} 4$ acetylation $(\mathrm{H} 4 \mathrm{ac})$ didn't alter greatly at the CREMa promoter. All these changes inhibited the expression of CREMa, then augmented IL-2 and down-modulated IL-17A productions. Subsequently, we observed that DNA methyltransferase (DNMT) 3a enrichment at the CREMa promoter was down-regulated significantly in SLE CD4+ T cells, and H3K4me3 amount was negatively correlated with both DNA methylation level and DNMT3a enrichment at the CREMa promoter in SLE CD4+ T cells.

Conclusions: In SLE CD4+ T cells, increased Set1 enrichment up-regulates H3K4me3 amount at the CREMa promoter, which antagonizes DNMT3a and suppresses DNA methylation within this region. All these factors induce CREMa overexpression, consequently result in IL-2 under-expression and IL-17A overproduction, and contribute to SLE at last. Our findings provide a novel approach in SLE treatment.
\end{abstract}

Keywords: Systemic lupus erythematosus, CREMa, H3K4me3, Set1, DNA methylation, DNMT3a

\footnotetext{
* Correspondence: qianlu5860@gmail.com

'Department of Dermatology, Second Xiangya Hospital, Central South

University, Changsha, Hunan 410011, China

Full list of author information is available at the end of the article
} 


\section{Background}

Systemic lupus erythematosus (SLE) is a chronic autoimmune disease which multiple pathogenic mechanisms are involved in [1, 2]. Recently, accumulating studies have documented that epigenetic alterations in certain genes of T cells play critical roles in the pathogenesis of SLE [3, 4]. Epigenetics refers to heritable changes in gene expression without changes in the DNA sequence $[5,6]$. The epigenetic mechanisms include mainly DNA methylation, histone modifications, noncoding RNA regulation, and chromatin modifications [5, 7]. It has been proved that DNA methylation is hallmark of gene silencing [8], while $\mathrm{H} 3$ lysine 4 trimethylation (H3K4me3), H3 acetylation (H3ac), and $\mathrm{H} 4$ acetylation (H4ac) are all correlated with transcriptional activation [9-11]. As one of the most familiar histone modifications, H3K4me3 is always a focus of epigenetics. It accumulates predominantly at the promoters and early transcribed regions of active genes, and is involved in transcription initiation, elongation and RNA processing by interacting with RNA polymerase II [12, 13]. It also can recruit and/or stabilize chromatin-remodeling enzymes and transcriptional cofactors [14, 15]. Interestingly, H3K4me3 is able to inhibit DNA methylation by antagonizing DNA methyltransferase (DNMT) 3a [16], and augment histone acetylation by interacting with histone acetyltransferases (HATs) [17]. As we all know, histone methyltransferases (HMTs) SET domain containing 1 (Set1) and mixed-lineage leukemia 1 (MLL1) can both catalyze trimethylation of H3K4 [18, 19].

Set1 and MLL1 are both large proteins containing one C-terminal SET domain that is associated with an intrinsic histone lysine-specific methyltransferase activity [20-22]. They are present, respectively, as the catalytic subunit and central element of multi-protein H3K4 methyltransferase complexes named complex of proteins associated with Set1 (COMPASS) and COMPASS-like [23-25]. Besides the catalytic Set1/MLL1 subunit, COMPASS/COMPASS-like contains several other proteins. Set1/MLL1 protein alone possesses very weak HMT activity, and their full activities require the context of the whole complexes [26, 27].

$\mathrm{T}$ cells from SLE patients and murine models produce less IL-2 compared to normal controls, and lower IL-2 level in SLE patients with higher SLE Disease Activity Index (SLEDAI) [28, 29]. Decreased IL-2 expression results in impaired generation of cytotoxic responses, reduced number and function of $\mathrm{T}$ regulatory cells (Tregs), and defective activation-induced cell death (AICD). In SLE patients, various cytotoxic responses have been reported ineffective and may account for the increased susceptibility to infection. The inhibited Tregs are unable to prevent autoimmunity, and the deficiency in AICD may lead to extended survival of autoreactive T cells, thereby B cells overactivate, in the end, resulting in overproduction of autoantibodies and the development of SLE [30-32].

Contrary to IL-2, T cells from patients with SLE and SLE murine models produce higher amounts of IL-17A, and IL-17A level is positively correlated with disease activity of SLE and titer of anti-dsDNA. Concordantly, inhibition of IL-17A can decrease the manifestations of lupus [33-36]. IL-17A is able to interact with various chemokines and cytokines, consequently triggers profound proinflammatory responses. It also stimulates B cells to proliferate and product more antibodies (including total IgG, anti-DNA and anti-histone antibodies) [28, 37, 38]. All these contribute to the onset of SLE.

Among the factors that regulate IL-2 and IL-17A, the cyclic adenosine 5'-monophosphate (cAMP) response element modulator $\alpha(\mathrm{CREM} \alpha)$ plays crucial roles in SLE. It has been reported that CREM $\alpha$ is increased in T cells from SLE patients, and the CREM $\alpha$ promoter activity is positively correlated with SLE disease activity [29]. The overexpression of CREM $\alpha$ can suppress TCR/CD3 $\zeta$ chain transcription, which is able to terminate the $\mathrm{T}$ cell response. It also represses the transcription factor c-fos, the antigen-presenting cell molecule CD86, and Notch signaling receptor Notch-1 to participate in the pathogenesis of SLE [35, 39-41]. And, the most important mechanism is that overexpressing CREM $\alpha$ can repress IL-2, yet increased IL-17A [32, 42]. However, which factors and mechanisms contribute to increased CREM $\alpha$ in SLE T cells remain unclear.

Through methylated CpG-DNA immunoprecipitation (MeDIP), Hedrich CM et al. found that DNA methylation level at the CREM $\alpha$ promoter in SLE CD $4^{+} \mathrm{T}$ cells is lower than healthy controls; moreover, CREM $\alpha$ promoter methylation is reduced in SLE patients who were in active stage compared to the patients in remission [29]. By chromatin immunoprecipitation (ChIP) microarray, we found that H3K4me3 enrichment at the CREM $\alpha$ promoter was significantly higher in SLE CD4 $4^{+}$ $T$ cells than in healthy controls. We then confirmed this result by ChIP and real-time PCR. In addition, a marked increase in Set1 binding was observed, but no striking change in MLL1 binding at the CREM $\alpha$ promoter in $\mathrm{CD} 4^{+} \mathrm{T}$ cells of patients with SLE. Knocking down Set1 with siRNA in SLE CD4 $4^{+} \mathrm{T}$ cells resulted in reduced both Set1 binding and H3K4me3 enrichment at the CREM $\alpha$ promoter, thus suppressing the expression of CREM $\alpha$, and increasing the amount of IL-2, simultaneously decreasing the production of IL-17A. We also found the levels of both DNA methylation and DNMT3a were elevated, while the concentrations of $\mathrm{H} 3 \mathrm{ac}$ and H4ac did not change greatly within the CREM $\alpha$ promoter in SLE CD4 ${ }^{+} \mathrm{T}$ cells whose Set1 was knocked down. According to this clue, we further verified that DNMT3a was decreased within the CREM $\alpha$ promoter in 
SLE CD4 ${ }^{+} \mathrm{T}$ cells, and H3K4me3 enrichment was negatively correlated with both DNA methylation level and DNMT3a binding at the promoter. Taken together, these results provide novel insights into the epigenetic mechanisms that cause SLE.

\section{Methods \\ Subjects}

Twenty SLE patients (age 27.10 \pm 6.52 years) were recruited from the out-patient clinics and in-patient wards of the Second Xiangya Hospital, Central South University, China. All patients fulfilled at least four of the SLE classification criteria of the American College of Rheumatology (ACR) [43]. Relevant clinical information of the SLE patients is listed in Table 1. Twenty healthy donors (age: $28.20 \pm 5.21$ years) were recruited from medic staff and graduate students at the Second Xiangya Hospital. All patients and controls were age- and sex-matched, and written informed consent was obtained from every participant. This study was approved by the Human Ethics Committee of the Central South University Second Xiangya Hospital and was conducted in accordance with the Declaration of Helsinki.

Table 1 Profiles of patients with SLE

\begin{tabular}{|c|c|c|c|c|}
\hline Patient & Gender & Age (years) & SLEDAI & Medications \\
\hline 1 & Female & 23 & 8 & Pred $30 \mathrm{mg} / \mathrm{d}$ \\
\hline 2 & Female & 20 & 6 & Pred $20 \mathrm{mg} / \mathrm{d}$ \\
\hline 3 & Male & 38 & 7 & Pred $20 \mathrm{mg} / \mathrm{d}$ \\
\hline 4 & Female & 21 & 3 & None \\
\hline 5 & Female & 26 & 12 & None \\
\hline 6 & Female & 28 & 12 & Pred $40 \mathrm{mg} / \mathrm{d}$ \\
\hline 7 & Female & 35 & 4 & HCQ $0.2 \mathrm{~g} / \mathrm{d}$ \\
\hline 8 & Female & 19 & 2 & None \\
\hline 9 & Female & 33 & 3 & Pred $5 \mathrm{mg} / \mathrm{d}$ \\
\hline 10 & Female & 27 & 2 & None \\
\hline 11 & Female & 32 & 15 & Pred $40 \mathrm{mg} / \mathrm{d}, \mathrm{TG}^{\mathrm{c}} 30 \mathrm{mg} / \mathrm{d}$ \\
\hline 12 & Female & 22 & 4 & HCQ $0.2 \mathrm{~g} / \mathrm{d}$ \\
\hline 13 & Female & 20 & 3 & Pred $5 \mathrm{mg} / \mathrm{d}$ \\
\hline 14 & Female & 22 & 10 & Pred $30 \mathrm{mg} / \mathrm{d}$, TG $30 \mathrm{mg} / \mathrm{d}$ \\
\hline 15 & Female & 25 & 0 & None \\
\hline 16 & Male & 40 & 10 & Pred $40 \mathrm{mg} / \mathrm{d}, \mathrm{HCQ} 0.2 \mathrm{~g} / \mathrm{d}$ \\
\hline 17 & Female & 30 & 16 & Pred $50 \mathrm{mg} / \mathrm{d}$, TG $30 \mathrm{mg} / \mathrm{d}$ \\
\hline 18 & Female & 26 & 2 & $\mathrm{HCQ} 0.2 \mathrm{~g} / \mathrm{d}$ \\
\hline 19 & Female & 20 & 8 & None \\
\hline 20 & Female & 35 & 12 & Pred 35 mg/d, HCQ $0.2 \mathrm{~g} / \mathrm{d}$ \\
\hline
\end{tabular}

SLEDAl systemic lupus erythematosus, Pred prednisone, HCQ hydroxychloroquine, $T G$ tripterygium glycoside

\section{Cell isolation}

Peripheral blood mononuclear cells (PBMCs) were isolated by Ficoll-Hypaque density gradient centrifugation (GE Healthcare), and $\mathrm{CD}^{+}{ }^{+} \mathrm{T}$ cells were subsequently isolated by positive selection using magnetic beads (Miltenyi), according to the manufacturer's instruction. The purity of enriched $\mathrm{CD} 4^{+} \mathrm{T}$ cells was generally higher than $95 \%$, as checked by flow cytometry.

\section{ChIP microarray}

$\mathrm{CD} 4^{+} \mathrm{T}$ cells from five SLE patients (relevant clinical information is listed in Additional file 1: Table S1) and five age- and sex-matched healthy controls were fixed with $1 \%$ formaldehyde for $10 \mathrm{~min}$, then they were lysed by lysis buffer. Lysates from SLE patients and healthy controls were pooled respectively, and were sent to Capitalbio (Beijing, China). ChIP microarray quality control, labeling, hybridization, scanning, and statistical analyze were carried out by Capitalbio. Anti-H3K4me3 antibodyprecipitated DNA and total DNA (input) were labeled with Cy5 (red) and Cy3 (green), respectively. Samples were then cohybridized onto the microarray panels, subsequently $\mathrm{Cy} 3 / \mathrm{Cy} 5$ ratio images of the microarray were generated. In these images, diversified color intensities represented relative $\mathrm{H} 3 \mathrm{~K} 4 \mathrm{me} 3$ enrichments at various gene promoters. Compared to control $\mathrm{CD}_{4}^{+} \mathrm{T}$ cells, at least twofold increase or decrease in H3K4me3 enrichments in SLE CD4 ${ }^{+} \mathrm{T}$ cells were considered significant.

\section{ChIP and real-time PCR}

ChIP assay was performed using a ChIP kit (Millipore), according to the instruction provided by the manufacturer. Briefly, $\mathrm{CD} 4^{+} \mathrm{T}$ cells were fixed with $1 \%$ formaldehyde for $10 \mathrm{~min}$, then lysed with lysis buffer. Cell lysates were sonicated to shear the DNA, subsequently the sonicated extracts were clarified by centrifugation. After preclearing by protein $\mathrm{G}$-agarose beads, antibodies were added and incubated with the extracts at $4{ }^{\circ} \mathrm{C}$ overnight on a rotator. The next day, protein G-agarose beads were added and rotated for $1 \mathrm{~h}$ at $4{ }^{\circ} \mathrm{C}$ to pull down immunoprecipitated complexes. The complexes were washed and subsequently eluted with elution buffer. After reversing cross links between DNA and protein by heating at $65{ }^{\circ} \mathrm{C}$ for $4 \mathrm{~h}$, the DNA was purified and subjected to real-time PCR analysis, and the input DNA was used as endogenous control. All experiments were performed three times. The primers for CREM $\alpha$ promoter were: forward 5 '-TGGGGAGATAGAGGTTGC AG-3' and reverse 5'-CGCCAGAAATCCAATGACTT3'. The anti-H3K4me3 antibody, anti-H3ac antibody, and anti-H4ac antibody were purchased from Millipore, and the anti-Set1 antibody, anti-MLL1 antibody, and anti-DNMT3a antibody were from Abcam. 
RNA extraction and real-time RT-PCR

Total RNA was isolated from $\mathrm{CD}^{+}{ }^{+} \mathrm{T}$ cells using TRIzol Reagent (Invitrogen) according to the protocol provided by the manufacturer, and stored at $-80{ }^{\circ} \mathrm{C}$. Real-time RT-PCR was performed with a Rotor-Gene3000 thermocycler (Corbett Research), and mRNA level was quantified by a SYBR PrimeScript RT-PCR kit (Takara). $\beta$-actin was amplified simultaneously as an endogenous control. Negative control (using water instead of RNA) was also run for every experiment. All reactions were run in triplicate. Primers used were as follows: for CREM $\alpha$, forward 5'-GAAACAGTTGAATCCCAGCATGATGGAAG T-3' and reverse 5'-TGCCCCGTGCTAGTCTGATAT ATG-3'; for $\beta$-actin, forward 5'-CGCGAGAAGATGACC CAGAT-3' and reverse 5'-GCACTGTGTTGGCGTACA GG-3'.

\section{Transfection}

Control-siRNA and Set1-siRNA were all designed and synthesized at Guangzhou RiboBio in China. SiRNA transfections were performed with a Human $\mathrm{T}$ Cell Nucleofector kit and a nucleofector (Amaxa), according to the protocols provided by the manufacturer. The transfected $\mathrm{CD}_{4}^{+} \mathrm{T}$ cells were then cultured in human $\mathrm{T}$ cell culture medium containing $10 \%$ fetal bovine serum (FBS). $24 \mathrm{~h}$ after transfection, the cells were stimulated with $5.0 \mu \mathrm{g} / \mathrm{ml}$ anti-CD3 and $2.5 \mu \mathrm{g} /$ $\mathrm{ml}$ anti-CD28 antibodies for $48 \mathrm{~h}$, in order to activate $\mathrm{CD}^{+} \mathrm{T}$ cells. Whereafter, they were subjected to further analysis.

\section{Western blotting}

$\mathrm{CD}_{4}^{+} \mathrm{T}$ cells were lysed with whole cell lysis buffer, and proteins were extracted and separated by SDSpolyacrylamide gel electrophoresis, then they were transferred to PVDF membranes (Millipore). The membranes were blocked in TBST buffer containing 5\% non-fat milk, and incubated overnight at $4{ }^{\circ} \mathrm{C}$ with CREM $\alpha$ antibody (1:500, Abcam), Set1 antibody (1:500, Abcam), or $\beta$-actin antibody (1:1000, Santa Cruz). All experiments were repeated three times, and relative expression levels were quantified by Quantity One software (Bio-Rad).

\section{ELISA}

IL-2 and IL-17A productions in the supernatants of stimulated $\mathrm{T}$ cells were measured by IL-2 and IL-17A quantification ELISA kits respectively (Yuanxiang), both following the manufacturer's instructions. Three replicate wells were used for every sample, and all experiments were performed three times. OD values were read at $450 \mathrm{~nm}$ for both IL-2 and IL-17A quantification.

\section{MeDIP and real-time PCR}

The methylated CpG-DNA immunoprecipitation assay was performed following the manufacturer's instruction (Abcam). Briefly, cells were lysed by lysis buffer, and DNA was sheared to fragments of $200-1000$ bp by sonication. After centrifuging, the clear supernatants were incubated with antibody for 5-methylcytosine or normal mouse IgG as the negative control. Subsequently, methylated CPG-DNA was released from immunoprecipitated complexes. After purifying, the DNA was subjected to real-time PCR analysis, with input DNA as endogenous control. All experiments were performed in triplicate.

\section{Statistical analysis}

Results were presented as mean $\pm \mathrm{SD}$. Values were compared by Student's $t$ test (paired $t$ test was used to compare data from different transfections, and two-group $t$ test was used to compare others). Correlations were measured by Pearson's correlation coefficient. $P$ values less than 0.05 were considered significant. All results were analyzed with SPSS 16.0 software (SPSS Inc.).

\section{Results}

Increased H3K4me3 enrichment at the CREMa promoter in SLE CD4 ${ }^{+} \mathrm{T}$ cells in the results of ChIP microarray

We first used ChIP microarray to measure H3K4me3 enrichments at various gene promoters in pooled $\mathrm{CD}^{+}{ }^{+} \mathrm{T}$ cell lysates from SLE patients and healthy controls. Based on the microarray results, out of the total 20,832 distinct gene promoters screened, 493 showed a greater than twofold difference in H3K4me3 enrichments between the two groups. Among these, H3K4me3 enrichment at the CREM $\alpha$ promoter in SLE CD4 ${ }^{+} \mathrm{T}$ cells was 2.48 times higher than in control $\mathrm{CD}_{4}^{+} \mathrm{T}$ cells (Fig. 1a, b).

\section{Increased H3K4me3 enrichment at the CREMa promoter in SLE CD4 ${ }^{+} \mathrm{T}$ cells}

In order to verify the finding of ChIP microarray, ChIP and real-time PCR were performed to measure H3K4me3 enrichment at the CREM $\alpha$ promoter in $C D 4^{+}$ $\mathrm{T}$ cells from 20 SLE patients and 20 healthy controls. Compared to healthy controls, H3K4me3 enrichment at the CREM $\alpha$ promoter was significantly increased in SLE $\mathrm{CD}^{+}{ }^{+} \mathrm{T}$ cells (Fig. 2a, Additional file 1: Table S2), consistent with our ChIP microarray result. We further carried out real-time RT-PCR to examine CREM $\alpha$ mRNA level in $\mathrm{CD}^{+} \mathrm{T}$ cells from SLE patients, and documented that H3K4me3 enrichment at the promoter was positively correlated with CREM $\alpha$ mRNA level in SLE CD4 $4^{+} \mathrm{T}$ cells (Fig. 2b). 


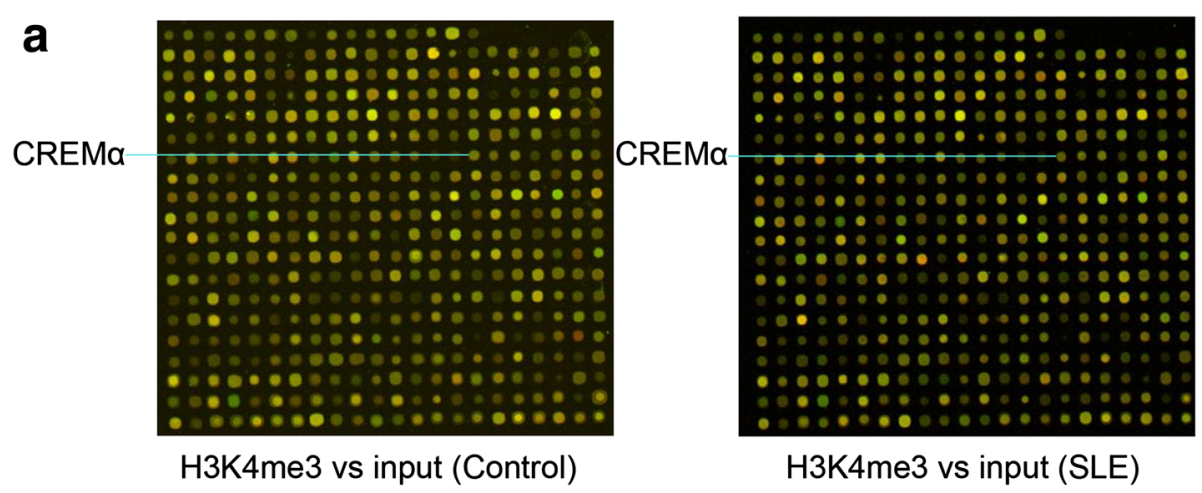

b

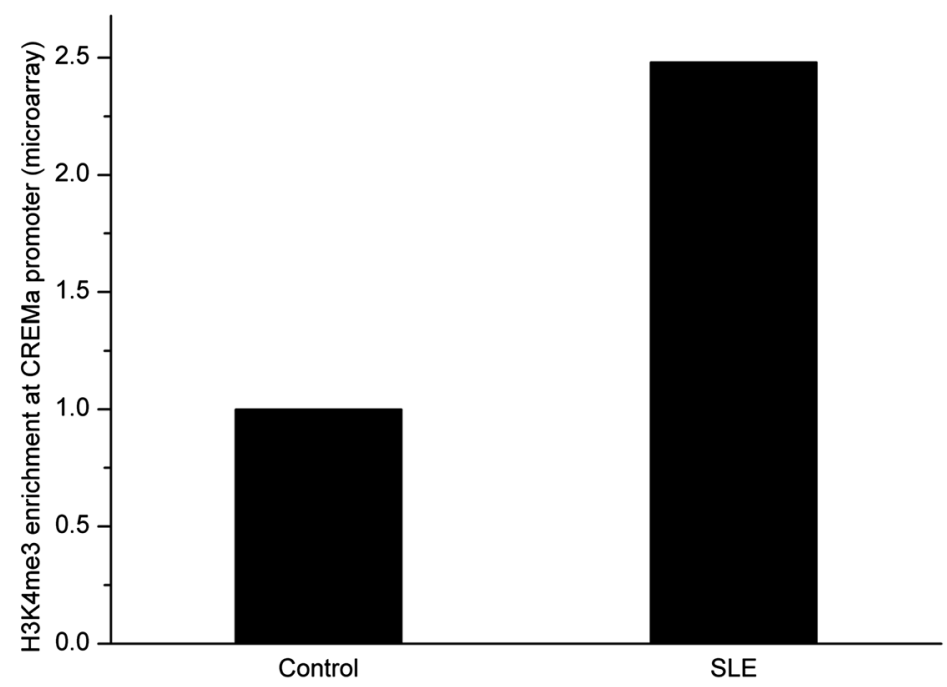

Fig. 1 ChIP microarray analysis of H3K4me3 enrichments in SLE and control CD4 ${ }^{+} \mathrm{T}$ cells. a ChIP microarray panels showing relative H3K4me3 enrichments at various gene promoters in $\mathrm{CD}^{+} \mathrm{T}$ cell lysates pooled from five healthy controls (left-hand panel) and five patients with SLE (right-hand panel). Anti-H3K4me3 antibody-precipitated DNA and total DNA (input) were respectively labeled with Cy5 (red) and Cy3 (green), and samples were subsequently cohybridized onto microarray panels. Each individual dot shows the Cy3/Cy5 ratio representing relative H3K4me3 enrichment at a specific gene promoter. The CREMa promoter dot (indicated by a blue line) is located in the sixteenth column, seventh row. b Relative H3K4me3 enrichment at the CREMa promoter in SLE and control CD4 ${ }^{+} \mathrm{T}$ cells, quantified from the results shown in (a)

Up-regulated Set1 binding at the CREMa promoter in SLE CD4 ${ }^{+} \mathrm{T}$ cells

Overexpression of H3K4me3 at the CREM $\alpha$ promoter in SLE CD4 ${ }^{+}$T cells prompted us to evaluate the status of two H3K4 methyltransferases, Set1 and MLL1. ChIP followed by real-time PCR was carried out to detect the levels of Set1 and MLL1 binding at the CREM $\alpha$ promoter in CD4 ${ }^{+}$ $\mathrm{T}$ cells from the 20 SLE patients and 20 healthy controls. A marked increase was identified in Set1 binding at the CREM $\alpha$ promoter in SLE CD4 $4^{+} \mathrm{T}$ cells compared with controls (Fig. 3a, Additional file 1: Table S2). However, MLL1 binding at the CREM $\alpha$ promoter did not demonstrate significant difference between SLE and control groups (Fig. 3b, Additional file 1: Table S2). In addition, we confirmed that the level of Set1 binding was positively correlated with both H3K4me3 enrichment at the CREM $\alpha$ promoter (Fig. 3c) and CREM $\alpha$ mRNA level in SLE CD4 ${ }^{+}$T cells (Fig. 3d).
Down-regulating Set1 in SLE CD4 ${ }^{+}$T cells inhibits CREMa expression

To confirm the effect of Set1 on CREM $\alpha$ expression, we transfected $\mathrm{CD}^{+} \mathrm{T}$ cells from three SLE patients with Set1-siRNA or control-siRNA. $72 \mathrm{~h}$ after transfection, total amounts of Set1 and CREM $\alpha$ were assessed by Western blotting. As expected, Set1 expression was sharply inhibited by Set1-siRNA compared to the control-siRNA group (Fig. 4a, b), and CREM $\alpha$ level was also downregulated significantly in $\mathrm{CD}^{+}{ }^{+} \mathrm{T}$ cells transfected with Set1-siRNA (Fig. 4a, b).

\section{Down-regulating Set 1 in SLE CD4 ${ }^{+} \mathrm{T}$ cells reduces $\mathrm{H} 3 \mathrm{~K} 4 \mathrm{me} 3$ enrichment at the promoter of CREMa}

In order to ascertain the mechanism whereby Set1 augments CREM $\alpha$ expression, we further analyzed Set1 and 

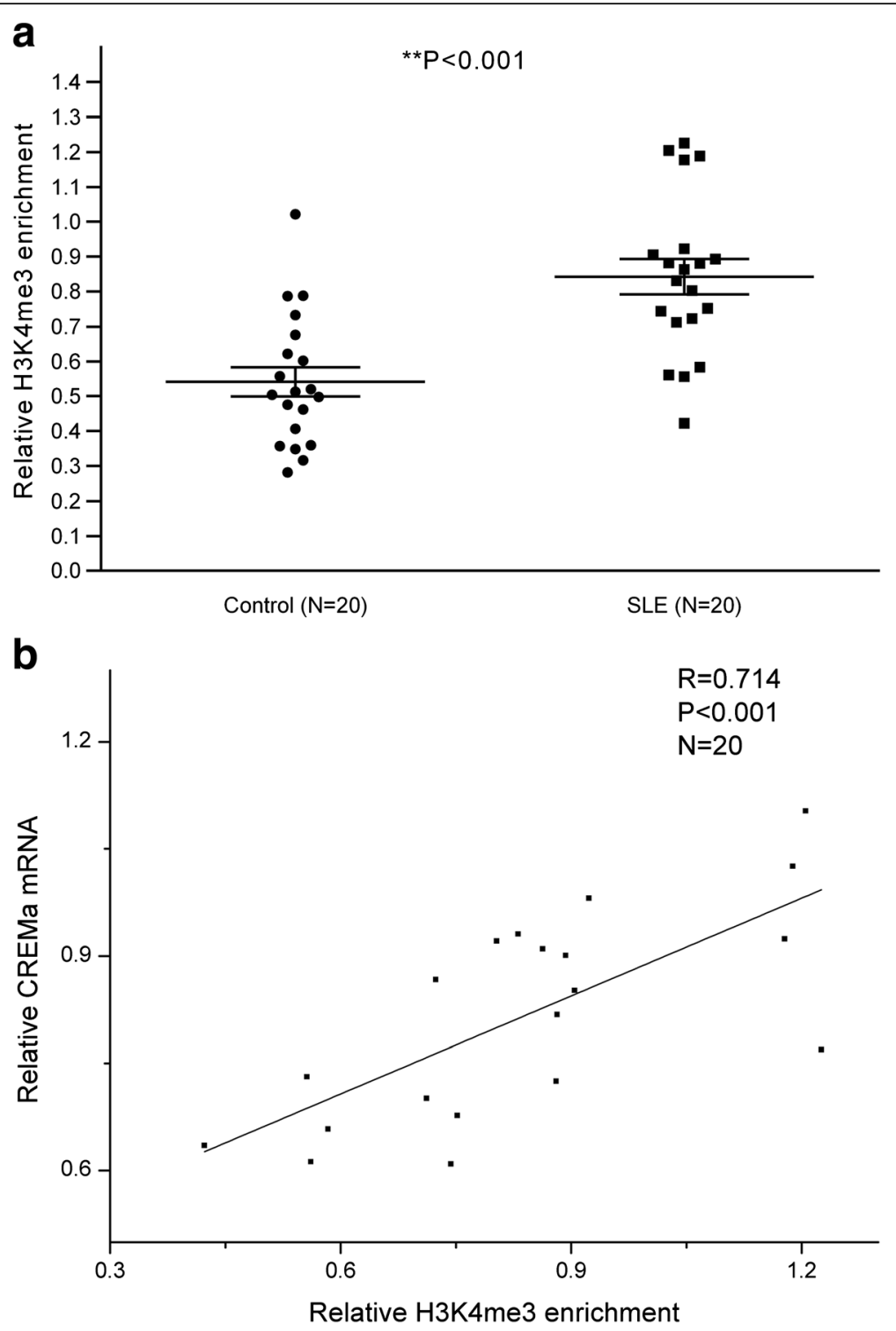

Fig. $2 \mathrm{H} 3 \mathrm{~K} 4 \mathrm{me} 3$ enrichment at the CREMa promoter in SLE and control CD4 ${ }^{+} \mathrm{T}$ cells. a Relative H3K4me3 enrichment within the CREMa promoter in SLE and healthy CD4 ${ }^{+} T$ cells was assessed by ChIP and real-time PCR. Results were normalized to input DNA (total chromatin). b Positive correlation between the levels of H3K4me3 and CREMa mRNA in SLE CD4 ${ }^{+} \mathrm{T}$ cells. All reactions were run in triplicate

H3K4me3 binding at the CREM $\alpha$ promoter in the aforementioned SLE CD4 ${ }^{+} \mathrm{T}$ cells by ChIP and real-time PCR. After transfection, Set1 binding at the CREM $\alpha$ promoter was also reduced together with total Set1 expression in the Set1-siRNA group (Fig. 4c). Concordantly, H3K4me3 level within the CREM $\alpha$ promoter was decreased after Set1 down-regulation (Fig. 4d).

\section{Down-regulating Set1 in SLE CD4 ${ }^{+}$T cells induces IL-2 and inhibits IL-17A}

Subsequently, we examined the effects of Set1 underexpression on IL-2 and IL-17A productions. $72 \mathrm{~h}$ after transfection, supernatant IL-2 and IL-17A concentrations of the SLE CD4 ${ }^{+}$T cells were measured by ELISA. Compared to control-siRNA group, we observed significantly increased IL-2 and deficient IL-17A in the supernatants collected from Set1-siRNA-transfected $\mathrm{CD}^{+} \mathrm{T}$ cells (Fig. 4e, f).

Down-regulating Set1 in SLE CD4 ${ }^{+} \mathrm{T}$ cells augments DNA methylation at the promoter of CREMa

It is well known that H3K4me3 can suppress DNA methylation and induce histone acetylation [11, 13, $16,44,45]$, so whether the changed H3K4me3 enrichment will alter the levels of DNA methylation and histone acetylation at the CREM $\alpha$ promoter in SLE 


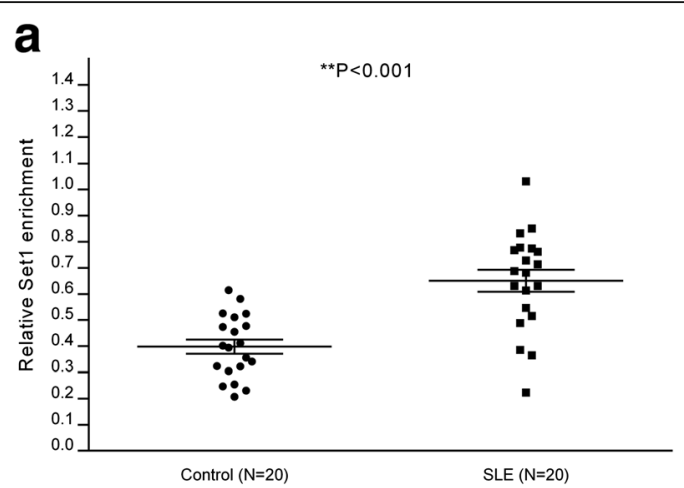

b
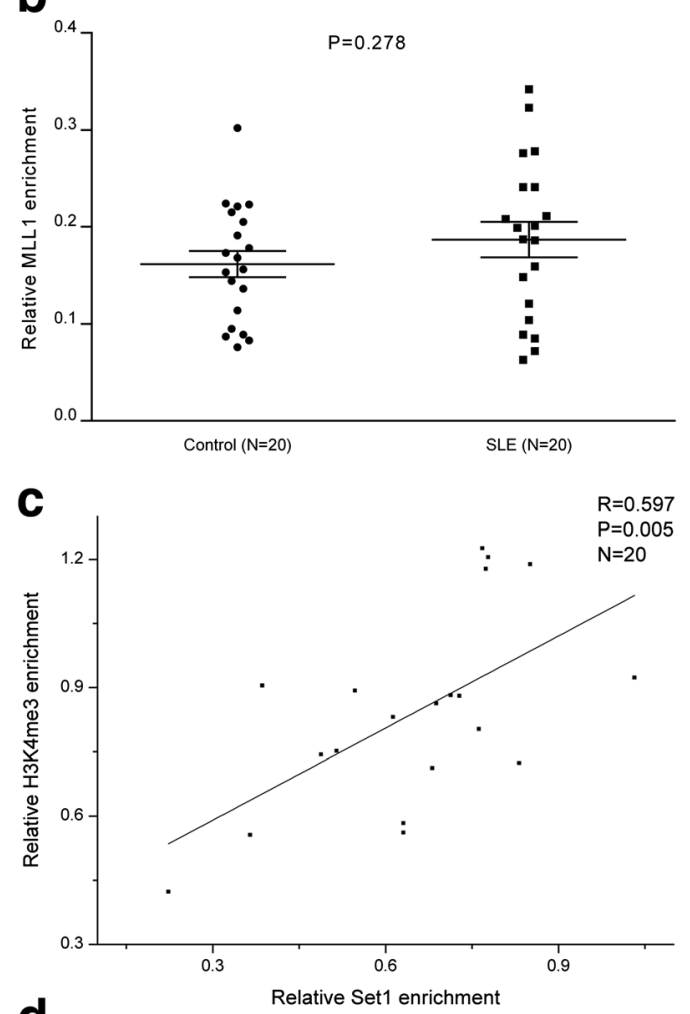

d

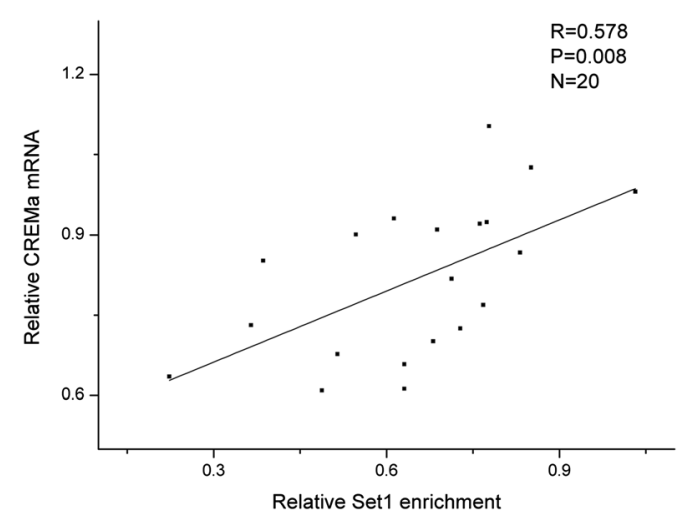

Fig. 3 Set1 and MLL1 binding at the CREMa promoter in SLE and control CD4 ${ }^{+} \mathrm{T}$ cells. $\mathbf{a}$, b Relative levels of Set1 (a) and MLL1 (b) binding within the CREMa promoter region in SLE and healthy CD4 ${ }^{+}$ T cells were analyzed by ChIP and real-time PCR. Results were normalized to input DNA (total chromatin). c Positive correlation between Set1 promoter binding and H3K4me3 level in SLE CD4 ${ }^{+} \mathrm{T}$ cells. $\mathbf{d}$ Positive correlation between Set1 promoter binding and CREMa mRNA level in SLE CD4 ${ }^{+}$T cells. All experiments were repeated three times

$\mathrm{CD}^{+} \mathrm{T}$ cells is still in question. We measured the quantity of DNA methylation by MeDIP and real-time $\mathrm{PCR}$, and detected the expressions of H3ac and H4ac by ChIP and real-time PCR in the siRNA-transfected SLE $\mathrm{CD}^{+} \mathrm{T}$ cells. Compared with the control-siRNA group, DNA methylation at the promoter of CREM $\alpha$ in the Set1siRNA group was upgraded greatly (Fig. 4g), in addition, $\mathrm{H} 3 \mathrm{ac}$ and H4ac enrichments at this region were both mildly decreased, but their changes were not significant (Fig. 4h, i).

\section{Down-regulating Set1 in SLE CD4 ${ }^{+} \mathrm{T}$ cells increases DNMT3a binding at the promoter of CREMa}

Since the quantity of DNA methylation is increased at the promoter of CREM $\alpha$ in these Set1-siRNA-transfected SLE $\mathrm{CD}^{+}{ }^{+} \mathrm{T}$ cells, we further assessed DNMT3a binding at the region with ChIP and real-time PCR. Consistent with our finding, the level of DNMT3a was elevated markedly in Set1-siRNA group (Fig. 4j).

\section{Negatively correlative $\mathrm{H} 3 \mathrm{~K} 4 \mathrm{me} 3$ enrichment and DNA methylation level at the CREMa promoter in SLE CD4 ${ }^{+}$ T cells}

Hedrich CM et al. have observed that DNA methylation level at the CREM $\alpha$ promter in SLE CD4 ${ }^{+} \mathrm{T}$ cells was lower than healthy controls [29]. In order to further investigate the relationship between $\mathrm{H} 3 \mathrm{~K} 4 \mathrm{me} 3$ and DNA methylation at the CREM $\alpha$ promoter in SLE CD4 ${ }^{+} \mathrm{T}$ cells, we examined the level of DNA methylation within the CREM $\alpha$ promoter in $\mathrm{CD}^{+} \mathrm{T}$ cells from the aforementioned 20 SLE patients via MeDIP and real-time $\mathrm{PCR}$, and proved that $\mathrm{H} 3 \mathrm{~K} 4 \mathrm{me} 3$ enrichment was negatively correlated with DNA methylation level at the CREM $\alpha$ promoter in SLE CD4. ${ }^{+}$T cells (Fig. 5a, Additional file 1: Table S2).

\section{Decreased DNMT3a binding at the CREMa promoter in SLE CD4 ${ }^{+} \mathrm{T}$ cells}

We further assayed the expression of DNMT3a within the CREM $\alpha$ promoter in $\mathrm{CD}_{4}^{+} \mathrm{T}$ cells from the aforementioned 20 SLE patients and 20 healthy controls by ChIP and real-time PCR. Consequently, we unraveled that DNMT3a binding at the CREM $\alpha$ promoter was decreased greatly in SLE CD4 ${ }^{+} \mathrm{T}$ cells (Fig. 5b, Additional 

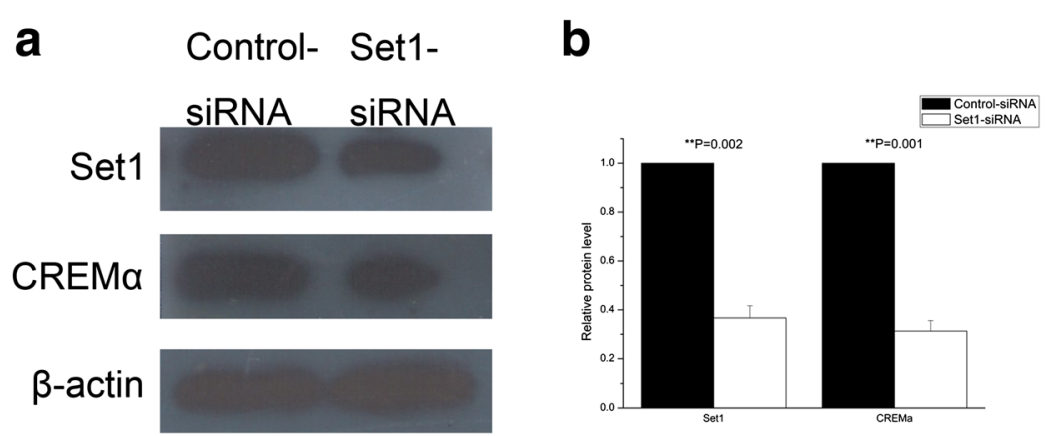

C

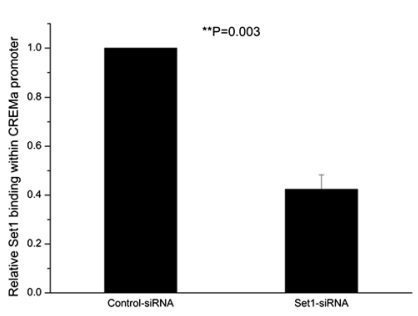

d

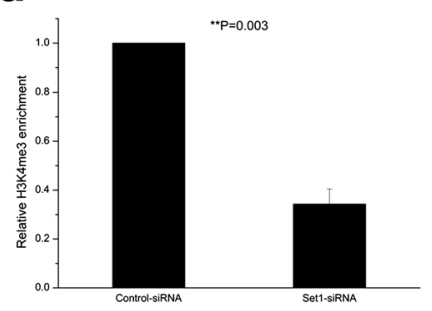

e

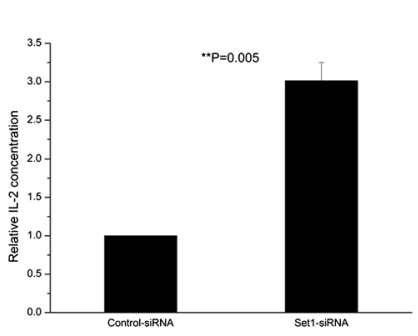

f

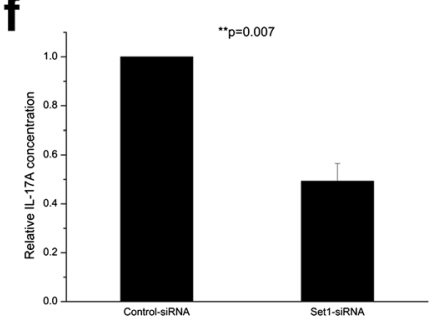

9

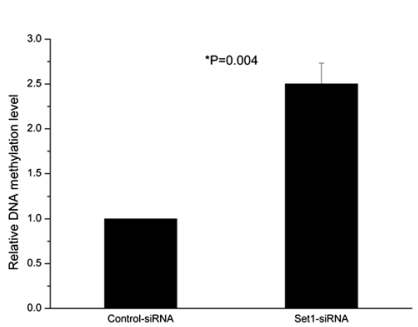

h

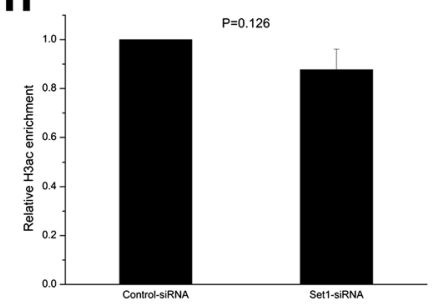

i

j

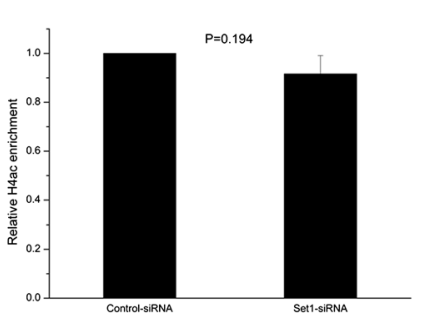

Fig. 4 (See legend on next page.)

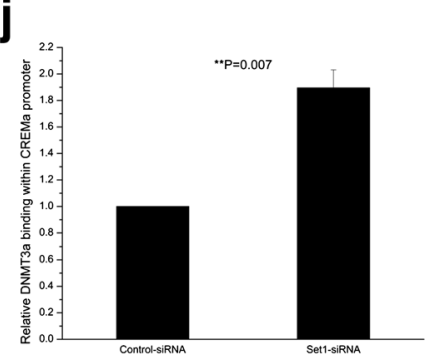




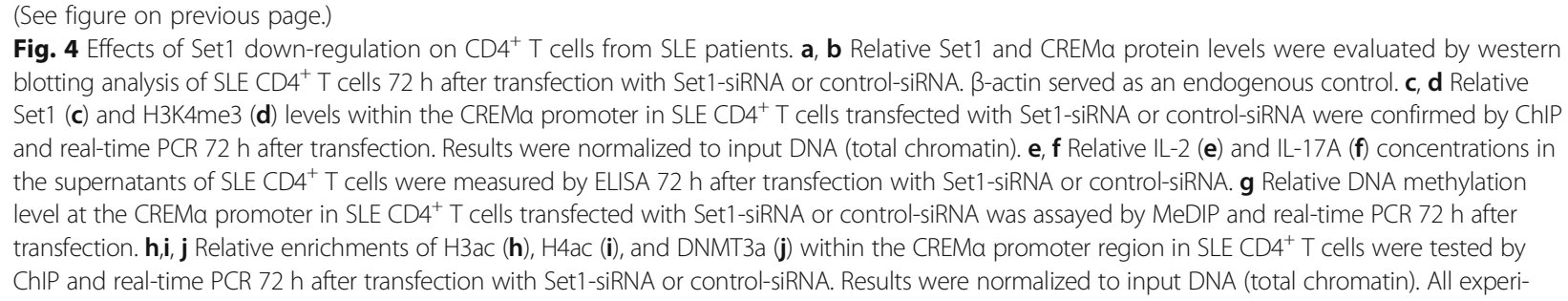

file 1: Table S2), and H3K4me3 enrichment was also negatively correlated with the amount of DNMT3a (Fig. 5c).

\section{Discussion}

In recent years, many researches have focused on the roles of CREM $\alpha$ in the pathogenesis of SLE, especially the mechanisms how CREM $\alpha$ inhibits IL-2 and induces IL-17A. However, the molecular mechanisms causing CREM $\alpha$ increasing in SLE T cells remain elusive.

By ChIP and real-time PCR, we confirmed our ChIP microarray finding that H3K4me3 enrichment at the CREM $\alpha$ promoter in SLE CD4 ${ }^{+} \mathrm{T}$ cells was significantly higher than in healthy controls. Furthermore, we documented that H3K4me3 enrichment was positively correlated with CREM $\alpha$ mRNA level. These data suggest that elevated H3K4me3 may be the cause of CREM $\alpha$ upregulation in SLE CD4 ${ }^{+} \mathrm{T}$ cells. We also proved that Set1 binding at the CREM $\alpha$ promoter was significantly increased in SLE CD4 ${ }^{+} \mathrm{T}$ cells, and Set1 binding was positively correlated with both H3K4me3 enrichment and CREM $\alpha$ mRNA level. However, there was no difference in MLL1 binding at the CREM $\alpha$ promoter between $\mathrm{CD}_{4}{ }^{+} \mathrm{T}$ cells from SLE patients and healthy controls. These findings suggest that it is not MLL1, but Set1 overproduction at the CREM $\alpha$ promoter that leads to H3K4me3 up-regulation, which in turn augments the expression of CREM $\alpha$.

Via siRNA-mediated knocking down, we observed that reducing Set1 in SLE CD4 ${ }^{+} \mathrm{T}$ cells down-regulated CREM $\alpha$ expression and Set1 binding at the CREM $\alpha$ promoter; accordingly, it decreased H3K4me3 enrichment within the same region, and increased IL-2 concentration, while inhibited IL-17A production. Together, these results indicate that Set1 regulates the expression of CREM $\alpha$, and this regulation is accomplished at least partly via changing H3K4me3 enrichment at the CREM $\alpha$ promoter; and the up-regulated Set1 binding at the promoter augments the generation of CREM $\alpha$ in SLE CD4 ${ }^{+}$T cells, subsequently results in IL-2 reduction and IL-17A overproduction. Since our manipulations not only altered the amount of Set1 at the CREM $\alpha$ promoter but also affected total Set1 level, we cannot eliminate the possibility that Set1 also regulates CREM $\alpha$, IL-2, and IL-17A in other ways.
In human, DNA can be methylated by DNMTs (including DNMT1, DNMT3a, and DNMT3b). In this process, DNMTs catalyze the methyl groups to the 5'carbon position of cytosine residues within CpG dinucleotides, forming 5-methylcytosine bases [8]. H3K4 methylation can down-regulate DNA methylation. It is reported DNMT3a recognizes the unmethylated H3K4 by its ADD domain, subsequently starts de novo DNA methylation [16]. In mutant strains whose H3K4 methylation is diminished, the DNA methylation expression increases fivefold [44]. H3K4me3 also interacts with inhibitor of growth family member 4 (ING4) of histone acetyltransferase binding to ORC-1 (HBO1) [10], Yng1 of NuA3 [46], Esa1 of NuA4 [17, 47], and chromoATPase/helicase-DNA binding domain 1 (Chd1) of SptAda-Gcn5 acetyltransferase (SAGA)/SAGA-like (SLIK) $[17,48]$, thereby recruits these HATs to target genes and enhances their HAT activity. In addition, H3K4me3 can disrupt binding of the nucleosome remodeling and deacetylase (NuRD) to $\mathrm{H} 3 \mathrm{~N}$-terminal tail, consequently preventing target gene deacetylation [49]. It is wellknown that DNA methylation can inhibit transcription of gene by changing the chromatin structure to a more compact and inactive form which blocks the access of some transcription factors [50]. On the contrary, histone acetylation can contribute to gene activation through relaxing the structure of chromatin [10, 11].

We have unraveled that H3K4me3 enrichment at the CREM $\alpha$ promoter was elevated in SLE $C D 4^{+} \mathrm{T}$ cells, therefore we further investigated whether the levels of DNA methylation, DNMT3a, H3ac, and H4ac at this region were affected by the alter of H3K4me3 in these SLE CD4 ${ }^{+} \mathrm{T}$ cells whose Set1 had been knocked down. We verified that both DNA methylation and DNMT3a at the promoter were up-regulated, while $\mathrm{H} 3 \mathrm{ac}$ and H4ac enrichments didn't change significantly.

Hedrich CM et al. have demonstrated that DNA methylation level at the CREM $\alpha$ promoter in SLE CD $4^{+}$ $\mathrm{T}$ cells was down-regulated [29], and our findings are consistent with their result. Taken together, all these data suggested that elevated H3K4me3 at the CREM $\alpha$ promoter excluded DNMT3a, which consequently limited DNA methylation at the same region in SLE CD4 ${ }^{+}$ $\mathrm{T}$ cells. In order to verify these conclusions, we 

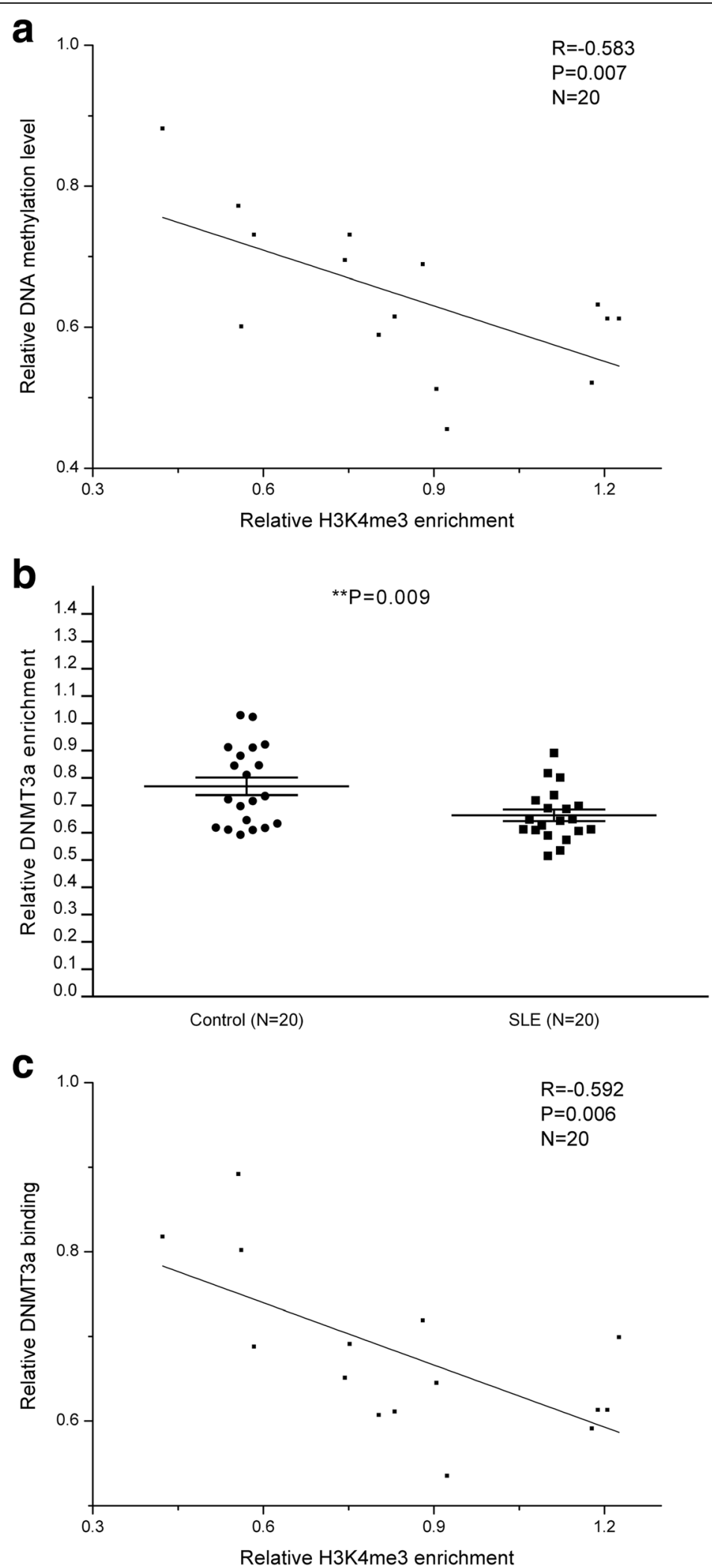

Fig. 5 (See legend on next page.) 
(See figure on previous page.)

Fig. 5 Relationships between H3K4me3, DNA methylation, and DNMT3a. a Negative correlation between H3K4me3 enrichment and DNA methylation level at the CREMa promoter in SLE CD4 ${ }^{+} T$ cells. $\mathbf{b}$ Relative level of DNMT3a binding within the CREMa promoter region in CD4 ${ }^{+} \mathrm{T}$ cells from 20 SLE patients and 20 healthy controls were detected by ChIP and real-time PCR. Results were normalized to input DNA (total chromatin). All data are representative from three independent experiments. c Negative correlation between H3K4me3 enrichment and DNMT3a binding at the CREMa promoter in SLE CD4 ${ }^{+}$T cells

measured the amounts of DNA methylation and DNMT3a within the CREM $\alpha$ promoter. As our expectation, DNMT3a was down-regulated greatly at the CREM $\alpha$ promoter in SLE CD4 ${ }^{+} \mathrm{T}$ cells compared to healthy controls; moreover, H3K4me3 enrichment was negatively correlated with both DNA methylation level and DNMT3a binding at the region in SLE CD4 ${ }^{+} \mathrm{T}$ cells.

\section{Conclusions}

Our results indicate that Set1 binding at the CREM $\alpha$ promoter is upgraded in SLE CD4 ${ }^{+} \mathrm{T}$ cells, and overexpressed Set1 up-regulates H3K4me3 level within the same region. Elevated H3K4me3 repels DNMT3a, and subsequently inhibited DNA methylation at the domain. All these contribute to CREM $\alpha$ overproduction, and consequently result in IL-2 increasing and IL-17A decreasing, ultimately causing the onset of SLE. Our findings indicate that the epigenetic mechanisms contribute to the development of SLE and provide a novel approach for the treatment of SLE.

\section{Additional file}

Additional file 1: Tables on profiles of SLE patients adopted in ChIP microarray and relevant results of SLE patients. Table S1. Profiles of SLE patients adopted in ChIP microarray. Table S2. Relevant results of SLE patients. (DOC $61 \mathrm{~kb})$

\begin{abstract}
Abbreviations
ACR: American College of Rheumatology; AICD: Activation-induced cell death; CAMP: Cyclic adenosine 5'-monophosphate; Chd1: Chromo-ATPase/ helicase-DNA binding domain 1; ChIP: Chromatin immunoprecipitation; COMPASS: Complex of proteins associated with Set1; CREMa: CAMP response element modulator a; DNMT: DNA methyltransferase; FBS: Fetal bovine serum; H3ac: H3 acetylation; H3K4me3: H3 lysine 4 trimethylation; H4ac: H4 acetylation; HAT: Histone acetyltransferase; HBO1: Histone acetyltransferase binding to ORC-1; HCQ: Hydroxychloroquine; HMT: Histone methyltransferase; ING4: Inhibitor of growth family member 4; MeDIP: Methylated CpG-DNA immunoprecipitation; MLL1: Mixed-lineage leukemia 1; NuRD: Nucleosome remodeling and deacetylase; PBMC: Peripheral blood mononuclear cell; Pred: Prednisone; SAGA: Spt-Ada-Gcn5 acetyltransferase; Set1: SET domain containing 1; SLE: Systemic lupus erythematosus; SLEDAl: SLE Disease Activity Index; SLIK: SAGA-like; TG: Tripterygium glycoside; Treg: T regulatory cell
\end{abstract}

\section{Acknowledgements}

Not applicable.

\section{Funding}

This work was supported by grants from the National Natural Science Foundation of China (No.81301359, No. 81220108017, No. 81430074, No. 81301357, and No. 81373205), the Ph.D. Programs Foundation of Ministry of Education of China (No. 20120162130003), the Hunan Provincial Natural Science Foundation of China (No. 14JJ1009), the Project of Innovation-driven Plan of Central South University (No. 2016CX029), and the National Key
Clinical Specialty Construction Project of National Health and Family Planning Commission of the People's Republic of China.

\section{Availability of data and materials}

The datasets are available from the corresponding author on reasonable request.

\section{Authors' contributions}

QZ conducted the sample collection, cell isolation, culture, transfection, ChIP, real-time PCR, RNA extraction, real-time RT-PCR, Western blotting, ELISA, MeDIP, statistical analysis, and drafted the manuscript. SD aided in sample collection and date interpretation. HLZ supervised sample collection and directed the statistical analysis. HL conducted the ChIP microarray and analysed its results. HJW helped with the manuscript writing and the final editing. MZ contributed to funding acquisition and manuscript revision. VC and CSL helped in editing and review of the manuscript. QJL designed the study, reviewed the data quality, helped with statistical analyses, and revised the manuscript. All authors read and approved the final manuscript.

\section{Competing interests}

The authors declare that they have no competing interests.

\section{Consent for publication}

Not applicable.

\section{Ethics approval and consent to participate}

This study was approved by the Human Ethics Committee of the Central South University Second Xiangya Hospital and was conducted in accordance with the Declaration of Helsinki. Written informed consent was obtained from every participant.

\section{Author details}

'Department of Dermatology, Second Xiangya Hospital, Central South University, Changsha, Hunan 410011, China. 'Department of Dermatology, Third Xiangya Hospital, Central South University, Changsha, Hunan 410011, China. ${ }^{3}$ Emergency Department, Second Xiangya Hospital, Central South University, Changsha, Hunan 410011, China. ${ }^{4}$ Division of Rheumatology and Clinical Immunology, Department of Medicine, The University of Hong Kong, Hong Kong, China.

Received: 5 August 2016 Accepted: 15 November 2016

Published online: 24 November 2016

\section{References}

1. Moulton VR, Holcomb DR, Zajdel MC, Tsokos GC. Estrogen upregulates cyclic AMP response element modulator alpha expression and downregulates interleukin-2 production by human T lymphocytes. Mol Med. 2012;18:370-8.

2. Tenbrock K, Kyttaris VC, Ahlmann M, Ehrchen JM, Tolnay M, Melkonyan H, et al. The cyclic AMP response element modulator regulates transcription of the TCR zeta-chain. J Immunol. 2005;175:5975-80.

3. Hewagama A, Richardson B. The genetics and epigenetics of autoimmune diseases. J Autoimmun. 2009;33:3-11.

4. Pan Y, Sawalha AH. Epigenetic regulation and the pathogenesis of systemic lupus erythematosus. Transl Res. 2009;153:4-10.

5. Lu Q, Renaudineau Y, Cha S, llei G, Brooks WH, Selmi C, et al. Epigenetics in autoimmune disorders: highlights of the 10th Sjogren's syndrome symposium. Autoimmun Rev. 2010;9:627-30.

6. Zhang Q, Long H, Liao J, Zhao M, Liang G, Wu X, et al. Inhibited expression of hematopoietic progenitor kinase 1 associated with loss of jumonji domain containing 3 promoter binding contributes to autoimmunity in systemic lupus erythematosus. J Autoimmun. 2011;37:180-9. 
7. Brooks WH, Le Dantec C, Pers JO, Youinou P, Renaudineau Y. Epigenetics and autoimmunity. J Autoimmun. 2010;34:J207-19.

8. Z Zhang P, Su Y, Lu Q. Epigenetics and psoriasis. J Eur Acad Dermatol Venereol. 2012;26:399-403.

9. Santos-Rosa H, Schneider R, Bannister AJ, Sherriff J, Bernstein BE, Emre $\mathrm{NC}$, et al. Active genes are tri-methylated at $\mathrm{K} 4$ of histone $\mathrm{H} 3$. Nature. 2002;419:407-11.

10. Hung T, Binda O, Champagne KS, Kuo AJ, Johnson K, Chang HY, et al. ING4 mediates crosstalk between histone $\mathrm{H} 3 \mathrm{~K} 4$ trimethylation and $\mathrm{H} 3$ acetylation to attenuate cellular transformation. Mol Cell. 2009;33:248-56.

11. Martin DG, Grimes DE, Baetz K, Howe L. Methylation of histone $\mathrm{H3}$ mediates the association of the NuA3 histone acetyltransferase with chromatin. Mol Cell Biol. 2006;26:3018-28.

12. Eissenberg JC, Shilatifard A. Histone H3 lysine 4 (H3K4) methylation in development and differentiation. Dev Biol. 2010;339:240-9.

13. Zhang $X$, Bernatavichute $Y$, Cokus S, Pellegrini M, Jacobsen SE. Genome-wide analysis of mono-, di- and trimethylation of histone H3 lysine 4 in Arabidopsis thaliana. Genome Biol. 2009;10:R62.

14. Iberg AN, Espejo A, Cheng D, Kim D, Michaud-Levesque J, Richard S, et al. Arginine methylation of the histone $\mathrm{H} 3$ tail impedes effector binding. J Biol Chem. 2008;283:3006-10.

15. Gregory GD, Vakoc CR, Rozovskaia T, Zheng X, Patel S, Nakamura T, et al. Mammalian ASH1L is a histone methyltransferase that occupies the transcribed region of active genes. Mol Cell Biol. 2007;27:8466-79.

16. Otani J, Nankumo T, Arita K, Inamoto S, Ariyoshi M, Shirakawa M. Structural basis for recognition of H3K4 methylation status by the DNA methyltransferase 3A ATRX-DNMT3-DNMT3L domain. EMBO Rep. 2009:10:1235-41.

17. Dehe PM, Geli V. The multiple faces of Set1. Biochem Cell Biol. 2006;84:536-48.

18. Wang $P$, Lin C, Smith ER, Guo H, Sanderson BW, Wu M, et al. Global analysis of H3K4 methylation defines MLL family member targets and points to a role for MLL1-mediated H3K4 methylation in the regulation of transcriptional initiation by RNA polymerase II. Mol Cell Biol. 2009;29:6074-85.

19. Yadav S, Singhal J, Singhal SS, Awasthi S. hSET1: a novel approach for colon cancer therapy. Biochem Pharmacol. 2009;77:1635-41.

20. Lee JS, Shukla A, Schneider J, Swanson SK, Washburn MP, Florens L, et al. Histone crosstalk between $\mathrm{H} 2 \mathrm{~B}$ monoubiquitination and $\mathrm{H} 3$ methylation mediated by COMPASS. Cell. 2007;131:1084-96.

21. Lee J, Saha PK, Yang QH, Lee S, Park JY, Suh Y, et al. Targeted inactivation of MLL3 histone H3-Lys-4 methyltransferase activity in the mouse reveals vital roles for MLL3 in adipogenesis. Proc Natl Acad Sc U S A. 2008:105:19229-34.

22. Lee JH, Skalnik DG. CpG-binding protein (CXXC finger protein 1) is a component of the mammalian Set1 histone H3-Lys4 methyltransferase complex, the analogue of the yeast Set1/COMPASS complex. J Biol Chem. 2005;280:41725-31.

23. Takahashi YH, Lee JS, Swanson SK, Saraf A, Florens L, Washburn MP, et al. Regulation of H3K4 trimethylation via Cps40 (Spp1) of COMPASS is monoubiquitination independent: implication for a Phe/Tyr switch by the catalytic domain of Set1. Mol Cell Biol. 2009;29:3478-86.

24. Wu M, Wang PF, Lee JS, Martin-Brown S, Florens L, Washburn M, et al. Molecular regulation of $\mathrm{H} 3 \mathrm{~K} 4$ trimethylation by Wdr82, a component of human Set1/COMPASS. Mol Cell Biol. 2008;28:7337-44.

25. Shilatifard A. Molecular implementation and physiological roles for histone $\mathrm{H} 3$ lysine $4(\mathrm{H} 3 \mathrm{~K} 4)$ methylation. Curr Opin Cell Biol. 2008;20:341-8.

26. Dehe PM, Dichtl B, Schaft D, Roguev A, Pamblanco M, Lebrun R, et al. Protein interactions within the Set1 complex and their roles in the regulation of histone 3 lysine 4 methylation. J Biol Chem. 2006:281:35404-12.

27. Issaeva I, Zonis Y, Rozovskaia T, Orlovsky K, Croce CM, Nakamura T, et al. Knockdown of ALR (MLL2) reveals ALR target genes and leads to alterations in cell adhesion and growth. Mol Cell Biol. 2007;27:1889-903.

28. Xu WD, Zhang YJ, Wang W, Li R, Pan HF, Ye DQ. Role of CREM in systemic lupus erythematosus. Cell Immunol. 2012;276:10-5.

29. Hedrich CM, Crispin JC, Rauen T, loannidis C, Apostolidis SA, Lo MS, et al. CAMP response element modulator alpha controls IL2 and IL 17A expression during CD4 lineage commitment and subset distribution in lupus. Proc Natl Acad Sci U S A. 2012;109:16606-11.
30. Gomez-Martin D, Diaz-Zamudio M, Crispin JC, Alcocer-Varela J. Interleukin 2 and systemic lupus erythematosus: beyond the transcriptional regulatory net abnormalities. Autoimmun Rev. 2009;9:34-9.

31. Kyttaris VC, Wang $Y$, Juang $Y T$, Weinstein A, Tsokos GC. CAMP response element modulator a expression in patients with systemic lupus erythematosus. Lupus. 2006;15:840-4.

32. Ohl K, Wiener A, Schippers A, Wagner N, Tenbrock K. Interleukin-2 treatment reverses effects of CAMP-responsive element modulator alpha-over-expressing $\mathrm{T}$ cells in autoimmune-prone mice. Clin Exp Immunol. 2015;181:76-86

33. Doreau A, Belot A, Bastid J, Riche B, Trescol-Biemont MC, Ranchin B, et al. Interleukin 17 acts in synergy with B cell-activating factor to influence B cell biology and the pathophysiology of systemic lupus erythematosus. Nat Immunol. 2009;10:778-85.

34. Hedrich CM, Rauen T, Kis-Toth K, Kyttaris VC, Tsokos GC. CAMP-responsive element modulator alpha (CREMalpha) suppresses IL-17 F protein expression in T lymphocytes from patients with systemic lupus erythematosus (SLE). J Biol Chem. 2012;287:4715-25.

35. Lippe R, Ohl K, Varga G, Rauen T, Crispin JC, Juang YT, et al. CREMalpha overexpression decreases IL-2 production, induces a $T(H) 17$ phenotype and accelerates autoimmunity. J Mol Cell Biol. 2012;4:121-3.

36. Rauen $\mathrm{T}$, Hedrich CM, Tenbrock K, Tsokos GC. CAMP responsive element modulator: a critical regulator of cytokine production. Trends Mol Med. 2013;19:262-9

37. Rauen T, Hedrich CM, Juang YT, Tenbrock K, Tsokos GC. CAMPresponsive element modulator (CREM)alpha protein induces interleukin $17 \mathrm{~A}$ expression and mediates epigenetic alterations at the interleukin17A gene locus in patients with systemic lupus erythematosus. J Biol Chem. 2011;286:43437-46.

38. Crispin JC, Tsokos GC. IL-17 in systemic lupus erythematosus. J Biomed Biotechnol. 2010:2010:943254.

39. Tenbrock K, Juang YT, Leukert N, Roth J, Tsokos GC. The transcriptional repressor CAMP response element modulator alpha interacts with histone deacetylase 1 to repress promoter activity. J Immunol. 2006;177:6159-64.

40. Rauen T, Grammatikos AP, Hedrich CM, Floege J, Tenbrock K, Ohl K, et al. CAMP-responsive element modulator alpha (CREMalpha) contributes to decreased notch-1 expression in T cells from patients with active systemic lupus erythematosus (SLE). J Biol Chem. 2012;287:42525-32.

41. Verjans E, Ohl K, Yu Y, Lippe R, Schippers A, Wiener A, et al. Overexpression of CREMalpha in T cells aggravates lipopolysaccharide-induced acute lung injury. J Immunol. 2013;191:1316-23.

42. Koga T, Hedrich CM, Mizui M, Yoshida N, Otomo K, Lieberman LA, et al. CaMK4-dependent activation of AKT/mTOR and CREM-alpha underlies autoimmunity-associated Th17 imbalance. J Clin Invest. 2014;124:2234-45.

43. Bombardier C, Gladman DD, Urowitz MB, Caron D, Chang CH. Derivation of the SLEDAI. A disease activity index for lupus patients. The Committee on Prognosis Studies in SLE. Arthritis Rheum. 1992;35:630-40.

44. Hu JL, Zhou BO, Zhang RR, Zhang KL, Zhou JQ, Xu GL. The N-terminus of histone $\mathrm{H} 3$ is required for de novo DNA methylation in chromatin. Proc Natl Acad Sci U S A. 2009:106:22187-92.

45. Hazzalin CA, Mahadevan LC. Dynamic acetylation of all lysine 4-methylated histone $\mathrm{H} 3$ in the mouse nucleus: analysis at c-fos and c-jun. PLoS Biol. 2005;3, e393.

46. Taverna SD, Ilin S, Rogers RS, Tanny JC, Lavender H, Li H, et al. Yng1 PHD finger binding to $\mathrm{H} 3$ trimethylated at $\mathrm{K} 4$ promotes NuA3 HAT activity at $\mathrm{K} 14$ of $\mathrm{H} 3$ and transcription at a subset of targeted ORFs. Mol Cell. 2006;24:785-96.

47. Morillon A, Karabetsou N, Nair A, Mellor J. Dynamic lysine methylation on histone $\mathrm{H} 3$ defines the regulatory phase of gene transcription. Mol Cell. 2005;18:723-34

48. Pray-Grant MG, Daniel JA, Schieltz D, Yates 3rd JR, Grant PA. Chd1 chromodomain links histone $\mathrm{H} 3$ methylation with SAGA- and SLIKdependent acetylation. Nature. 2005;433:434-8.

49. Zegerman P, Canas B, Pappin D, Kouzarides T. Histone H3 lysine 4 methylation disrupts binding of nucleosome remodeling and deacetylase (NuRD) repressor complex. J Biol Chem. 2002;277:11621-4.

50. Li Y, Sawalha AH, Lu Q. Aberrant DNA methylation in skin diseases. J Dermatol Sci. 2009:54:143-9. 